\title{
Stabilization of the variable-length pendulum
}

Link to publication record in Manchester Research Explorer

\section{Citation for published version (APA):}

Navarro Lopez, E., Navarro-Lopez, E. M., \& Fossas-Colet, E. (2001). Stabilization of the variable-length pendulum. In European Control Conference (pp. 675-680) http://www.cs.man.ac.uk/ navarroe/papers/enavarro_ecc01.pdf

\section{Published in:}

European Control Conference

\section{Citing this paper}

Please note that where the full-text provided on Manchester Research Explorer is the Author Accepted Manuscript or Proof version this may differ from the final Published version. If citing, it is advised that you check and use the publisher's definitive version.

\section{General rights}

Copyright and moral rights for the publications made accessible in the Research Explorer are retained by the authors and/or other copyright owners and it is a condition of accessing publications that users recognise and abide by the legal requirements associated with these rights.

\section{Takedown policy}

If you believe that this document breaches copyright please refer to the University of Manchester's Takedown Procedures [http://man.ac.uk/04Y6Bo] or contact uml.scholarlycommunications@manchester.ac.uk providing relevant details, so we can investigate your claim.

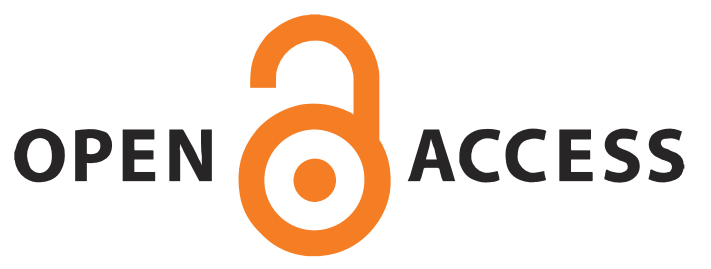




\title{
STABILIZATION OF THE VARIABLE-LENGTH PENDULUM
}

\author{
E.M. Navarro-López, E. Fossas-Colet ${ }^{\dagger}$ \\ Institut d'Organització i Control \\ Universitat Politècnica de Catalunya \\ Avda. Diagonal 647, 11 planta \\ 08028 BARCELONA, Spain \\ fax: +34-93-4016605 \\ e-mail: enavarrodiri.upc.es, fossas@ioc.upc.es
}

Keywords: Dissipative and Energy-based Design, Stabilization of Non-linear systems, Variable-length pendulum

\begin{abstract}
The variable-length pendulum, proved to be non-minimum phase and non static-state-feedback linearizable, is stabilized around an equilibrium point by means of the passivity-based design control technique of Energy Shaping plus Damping Injection
\end{abstract}

\section{Introduction}

The stabilization and study of systems stability properties by means of their energy characteristics have received a growing attention in systems theory. The concepts of passivity and dissipativity have had an important role in this fact. A passive system is such a system which cannot store all the amount of energy that has been given, i.e., it dissipates energy in some way. This definition implies the existence of a storage function (representing the energy stored by the system) and a supply function (external energy received by the system). The idea of stored energy can be used connected to the system stability, considering the stored energy function as a Lyapunov-like function. Indeed, this fact can be deduced directly from the fact that a passive system has the Kalman-Yakubovich-Popov (KYP) property [1].

Furthermore, if a system is passive, properties referring to its zero dynamics can be followed: passive systems are minimum phase, since if the system output is rendered zero by means of an adequate feedback, the remaining dynamics or zero dynamics is Lyapunov-stable.

The control of a system in terms of the energy it can store or dissipate is known as Passivity-Based Control (PBC), and gives us a rather intuitive approach exploiting the system physical properties and the interconnection of the system with its environment. The aim of passivity-based control design methodology is modifying the closed-loop system energy and the addition of the required dissipation, it is the Energy Shaping plus Damping Injection (ESDI) control technique.

\footnotetext{
${ }^{*}$ Under fellowship from Ministerio de Educación y Cultura and Consejo Superior de Investigaciones Científicas (España)

${ }^{\dagger}$ Corresponding author
}

Passivity concepts will make possible to distinguish different parts or components of the systems dynamics. Depending the stored energy of the system along the trajectories of the system decreases, increases or remains we will speak of the dissipative, non-dissipative or invariant (also called lossless) part of the system, respectively.

We will use passivity concepts in connection to the stabilization problem. We aim to stabilize the variable-length pendulum trajectories around a desired equilibria by means of an energy approach. System passivity properties will be used, and the Lyapunov's stability of the system equilibrium point will be guaranteed by means of assuring the existence of a Lyapunovlike energy function.

There are a lot of works relating the control of the variablelength pendulum. A first approach to the problem in the frame of Lagragian dynamics is the one given by Bressand et al [2]. In addition to the tracking problem for this particular system recently presented by Sira-Ramírez [14], we could also point out the solution to the regulation problem presented by Fliess et al [3].

The application of the passivity approach to systems control is found in a great variety of papers written in the last decades. There are mainly devoted to mechanical (such as robots), electro-mechanical (such as induction motors) and purely electrical systems (such as dc-to-dc power converters), for a survey see Ortega et al [9]. Some examples of application to robotics and Lagragian systems are the works of Ortega et al $[8,11]$ and the ones presented by Kelly and coworkers (for example [4]). Electro-mechanical systems were addressed, among others, in Nicklasson et al [7]. Passivity-based controllers for power electronics were given, for instance, in Sira-Ramírez et al [12]. Passivity-based control design has also been applied to a great variety of nonlinear systems.

The control design methodology to use will be the ESDI one, which consists of modifying the stored energy of the system for the desired equilibrium to be the minimum of the new energy function for the closed-loop system and the addition of the required dissipation. Generalities and details of this technique can be found in Ortega et al [10] and the work of Sira-Ramírez et al [12]. In the literature, the ESDI idea has been applied in two different ways, the main differences between them are related to the way the Energy Shaping is made. On the one hand, we have the approach of Ortega et al [11] and Maschke 
et al [6], for which the expression for the closed-loop system Lyapunov-like energy function will not be needed to be known in order to ensure the stability of the system equilibrium point. On the other hand, the work of Sira-Ramírez et al $[13,15]$ is based on the explicit knowledge of the expression for the system energy. Indeed, the energy shaping is made in a different way in the two approaches. In the first one, the new energy function is conceived as a variation of the initial one $(V+\Delta V$, considering $V$ the initial energy function), on the contrary, in the second one, the energy shaping is represented by the energy associated to the error system, which definition is based on the proposal of an auxiliary dynamics. We can also detect a difference between the two mentioned approaches through the purpose of the dissipation injected to the system.

This work follows the ESDI approach which does not require the knowledge of the new Lyapunov-like energy function. Section 2 presents a model for the variable-length pendulum. In section 3, we will apply the ESDI methodology for the studied system and derive a controller. The study of the properties of the resulting closed-loop system is dealt with in section 4 . Some simulations results, the conclusions and suggestions for further research are presented in the last two sections.

\section{The variable-length pendulum}

Consider the normalized model of the variable-length pendulum studied by Bressand and Rampazzo [2], given by

$$
\begin{aligned}
\dot{q} & =p \\
\dot{p} & =-\cos u+q v^{2} \\
\dot{u} & =v
\end{aligned}
$$

where $q$ denotes the distance from the center of the sliding ball to the origin of coordinates around which the rod rotates. The variable $p$ is the velocity of the ball, as measured along the rod, and $u$ is the angle formed by the rod and the vertical line passing through the origin (see figure 1 ). The angular velocity $v$ will be considered as the control.

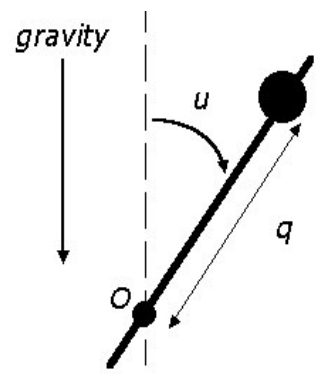

Figure 1: The inverted variable-length pendulum.

This system has interesting properties which make its stabilization a very attractive problem. We can check this system to be non-feedback linearizable by static state feedback and, hence, it is not differentially flat.

As it is proposed in Fliess [3], we will use an averaged model for the variable-length pendulum, the relation between the new model and the one presented in (1) will be

$$
v=v_{1}+v_{2} \cos (t / \varepsilon)
$$

where $v_{1}$ and $v_{2}$ are auxiliary controls and $\varepsilon$ is a constant $0<\varepsilon \ll 1$. Then, substituting (2) in (1) and considering the averaged control systems, it is obtained

$$
\begin{aligned}
\dot{\bar{q}} & =\bar{p} \\
\dot{\bar{p}} & =-\cos \bar{u}+\bar{q} v_{1}^{2}+\bar{q} \frac{v_{2}^{2}}{2} \\
\dot{\bar{u}} & =v_{1}
\end{aligned}
$$

Controls $v_{1}$ and $v_{2}$ are supposed to depend on the averaged states variables in a continuous and differentiable way.

The above input redefinition and the use of the averaged model overcome the drawback for the original system (1) to be nonflat.

\section{ESDI-based stabilization}

\subsection{Stabilization of the variable-length pendulum}

The ESDI approach is proved to stabilize the variable-length pendulum around a desired equilibrium point. Consider the dynamics (3) and the energy function for the system as $V$, we have

$$
V=\bar{q} \cos \bar{u}+\frac{1}{2} \bar{p}^{2}
$$

which gradient takes the following expression

$$
\frac{\partial V}{\partial \bar{x}}=(\cos \bar{u}, \bar{p},-\bar{q} \sin \bar{u})
$$

where $\bar{x}$ is the averaged state vector $\left(\bar{x}=(\bar{q}, \bar{p}, \bar{u})^{T}\right)$. Thus, system (3) can be rewritten as

$$
\begin{aligned}
\left(\begin{array}{c}
\dot{\bar{q}} \\
\dot{\bar{p}} \\
\dot{\bar{u}}
\end{array}\right) & =\left(\begin{array}{ccc}
0 & 1 & 0 \\
-1 & 0 & 0 \\
0 & 0 & 0
\end{array}\right)\left(\frac{\partial V}{\partial \bar{x}}\right)^{T}+ \\
& +\left(\begin{array}{c}
0 \\
\bar{q}\left(v_{1}^{2}+\frac{1}{2} v_{2}^{2}\right) \\
v_{1}
\end{array}\right)= \\
& =J(\bar{x})\left(\frac{\partial V}{\partial \bar{x}}\right)^{T}+D\left(\bar{x}, v_{1}, v_{2}\right)
\end{aligned}
$$

$J(\bar{x})$ is the $n \times n$-skew-symmetric matrix and $J(\bar{x})\left(\frac{\partial V}{\partial \bar{x}}\right)^{T}$ represents the invariant component of the system, $D$ is a vector field grouping the dissipative and non-dissipative terms for the system dynamics. For stability purposes, we would like to convert the terms appearing in $D$ into passive ones for some energy function, which may be different from the initial one $V$. Hence, the stabilization of the variable-length pendulum for an equilibrium point $\bar{x}^{*}$ will consist of finding controls $v_{1}=\beta_{1}(\bar{x})$ 
and $v_{2}=\beta_{2}(\bar{x})$ such that the system dynamics can be expressed as

$$
\left(\begin{array}{c}
\dot{\bar{q}} \\
\dot{\bar{p}} \\
\dot{\bar{u}}
\end{array}\right)=[J(\bar{x})-R(\bar{x})]\left(\frac{\partial V^{T}}{\partial \bar{x}}+\frac{\partial \Delta V^{T}}{\partial \bar{x}}\right),
$$

where $R(\bar{x})$ an $n \times n$-positive-semi-definite symmetric matrix.

Now, $J(\bar{x})\left[\frac{\partial(V+\Delta V)}{\partial \bar{x}}\right]^{T}$ represents the invariant component of the closed-loop system and, the dissipative one, that is, the one which implies a loss of energy as time goes forward, is given by $R(\bar{x})\left[\frac{\partial(V+\Delta V)}{\partial \bar{x}}\right]^{T}$.

$V_{d}=V+\Delta V$ is the new Lyapunov-like function to be used in order to ensure the stability of the system around the equilibrium point $\bar{x}^{*}=\left(\bar{q}^{*}, 0, \bar{u}^{*}\right)^{T}$.

Remark 3.1 $V_{d}$ is certainly a Lyapunov-like function. The function $\Delta V$ will be chosen in such a way for $\bar{x}^{*}$ to be a strict local minimum of $V_{d}$, i.e., there exists an open neighbourhood $\overline{\mathscr{X}}$ of $\bar{x}^{*}$ such that $V_{d}\left(\bar{x}^{*}\right)<V_{d}(\bar{x}), \forall \bar{x} \in \overline{\mathscr{X}}$. This condition is met whenever the derivative of $V_{d}$ with respect to $\bar{x}$ has an isolated zero at $\bar{x}^{*}$ and in addition to, the hessian of $V_{d}$ is a positive-definite quadratic form, namely

$$
\begin{aligned}
& \left(\frac{\partial V_{d}}{\partial \bar{x}}\right)_{\bar{x}=\bar{x}^{*}}=0 \\
& \left(\frac{\partial V_{d}}{\partial \bar{x}}\right)_{\bar{x} \neq \bar{x}^{*}} \neq 0 \\
& \left(\frac{\partial^{2} V_{d}}{\partial \bar{x}^{2}}\right)_{\bar{x}=\bar{x}^{*}} \text { be positive-definite }
\end{aligned}
$$

Moreover, the derivative of $V_{d}$ along the trajectories of the desired dynamics (6) takes the form,

$$
\dot{V}_{d}=\frac{\partial V_{d}}{\partial \bar{x}}[J(\bar{x})-R(\bar{x})]\left(\frac{\partial V_{d}}{\partial \bar{x}}\right)^{T}
$$

Since $\frac{\partial V_{d}}{\partial \bar{x}} J(\bar{x})\left(\frac{\partial V_{d}}{\partial \bar{x}}\right)^{T}=0$, then

$$
\dot{V}_{d}=-\frac{\partial V_{d}}{\partial \bar{x}} R(\bar{x})\left(\frac{\partial V_{d}}{\partial \bar{x}}\right)^{T} \leq 0
$$

Thus, we can assure that $\bar{x}^{*}$ is a locally stable equilibrium point for (6).

Expression (5) together with (6) leads us to

$$
\begin{aligned}
\left(\frac{\partial \Delta V}{\partial \bar{x}}\right)^{T} & =[J(\bar{x})-R(\bar{x})]^{-1}\left[R(\bar{x})\left(\frac{\partial V}{\partial \bar{x}}\right)^{T}+\right. \\
& \left.+D\left(\bar{x}, v_{1}, v_{2}\right)\right]
\end{aligned}
$$

We will design $R$ for expression (10) to be met, and for this purpose we need not know the expression for $\Delta V$, it is enough to know it exists.
The controller design procedure will be based on assuring the solvability of (10) and the fact that $\bar{x}^{*}$ is a strict local minimum of the energy function $V_{d}$ (7)-(9).

\subsubsection{Controller design}

For simplicity's sake, dynamics (3) will be rewritten as

$$
\begin{aligned}
\dot{\bar{q}} & =\bar{p} \\
\dot{\bar{p}} & =-\cos \bar{u}+\bar{q} w \\
\dot{\bar{u}} & =v_{1}
\end{aligned}
$$

where $w=v_{1}^{2}+\frac{1}{2} v_{2}^{2}, w>0$. Thus, the original multi-input averaged system of the general form $\dot{\bar{x}}=f(\bar{x}, u)$ has now an affinein-the-input structure $\dot{x}=f(\bar{x})+G(\bar{x}) u$

$$
\dot{\bar{x}}=\left(\begin{array}{c}
\bar{p} \\
-\cos (\bar{u}) \\
0
\end{array}\right)+\left(\begin{array}{ll}
0 & 0 \\
\bar{q} & 0 \\
0 & 1
\end{array}\right)\left(\begin{array}{l}
w \\
v_{1}
\end{array}\right)
$$

Choosing as $R$ the matrix

$$
R=\left(\begin{array}{ccc}
0 & 0 & 0 \\
0 & 0 & 0 \\
0 & 0 & r_{3}(\bar{q}, \bar{p}, \bar{u})
\end{array}\right),
$$

and taking into account the relation (10), we have for system dynamics (12)

$$
\frac{(\partial \Delta V)}{\partial \bar{x}}=\left(-\bar{q} w, 0, \bar{q} \sin \bar{u}-\frac{v_{1}}{r_{3}}\right)
$$

The existence of such a local function $\Delta V$, which gradient is the one written in (13), is assured if the rotational of $\Delta V$ is zero. This lead us to the following three conditions,

$$
\frac{\partial}{\partial \bar{p}}\left(\frac{v_{1}}{r_{3}}\right)=0 \Rightarrow \frac{v_{1}}{r_{3}}=\phi(\bar{q}, \bar{u})
$$

$$
\begin{gathered}
\sin \bar{u}-\frac{\partial}{\partial \bar{q}}\left(\frac{v_{1}}{r_{3}}\right)+\bar{q} \frac{\partial w}{\partial \bar{u}}=0 \\
\frac{\partial w}{\partial \bar{p}}=0 \Rightarrow w=w(\bar{q}, \bar{u})
\end{gathered}
$$

Putting together (14) and (16) into (15), we have

$$
v_{1}=r_{3} \phi(\bar{q}, \bar{u}), \omega=\omega(\bar{q}, \bar{u}) \text { and } \sin \bar{u}-\frac{\partial \phi}{\partial \bar{q}}+\bar{q} \frac{\partial w}{\partial \bar{u}}=0
$$

In order to fulfill (17), let us define

$$
\begin{aligned}
w & =\frac{\cos \bar{u}}{\bar{q}}+w_{1}(\bar{q}, \bar{u}) \\
w_{1} & =\frac{1}{\bar{q}}\left[a_{1}\left(\bar{q}-\bar{q}^{*}\right)+a_{2}\left(\bar{u}-\bar{u}^{*}\right)\right] \\
v_{1} & =a_{2} r_{3}\left(\bar{q}-\bar{q}^{*}\right)+a_{3}\left(\bar{u}-\bar{u}^{*}\right)
\end{aligned}
$$


where $a_{1}, a_{2}, a_{3}, r_{3}$ are constants. Then, we have

$$
\begin{aligned}
\frac{\partial(\Delta V)}{\partial \bar{x}}= & \left(-\bar{q} w, 0, \bar{q} \sin \bar{u}-\frac{v_{1}}{r_{3}}\right)= \\
= & \left(-\cos \bar{u}-a_{1}\left(\bar{q}-\bar{q}^{*}\right)-a_{2}\left(\bar{u}-\bar{u}^{*}\right), 0,\right. \\
& \left.\bar{q} \sin \bar{u}-a_{2}\left(\bar{q}-\bar{q}^{*}\right)-\frac{a_{3}}{r_{3}}\left(\bar{u}-\bar{u}^{*}\right)\right)
\end{aligned}
$$

It can be checked that conditions (7)- (9) are met, i.e. the equilibrium point $\left(\bar{q}^{*}, 0, \bar{u}^{*}\right)$ is an isolated strict local minimum for the closed-loop system energy function $V_{d}$ if and only if,

$$
\begin{aligned}
& a_{1}<0, a_{3}<0 \\
& a_{1} a_{3}-r_{3} a_{2}^{2}>0
\end{aligned}
$$

Note that $w$ is an auxiliary control used for simplifying the averaged model (3). We need the expression of $v_{1}$ and $v_{2}$ for the computation of the control $v$, thus, from

$$
v_{1}^{2}+\frac{v_{2}^{2}}{2}=w(\bar{q}, \bar{u})=\frac{\cos \bar{u}}{\bar{q}}+w_{1}(\bar{q}, \bar{u})
$$

the control $v_{2}$ can be obtained,

$$
v_{2}=\sqrt{2\left(\frac{\cos \bar{u}}{\bar{q}}+w_{1}-v_{1}^{2}\right)}
$$

where

$$
\begin{aligned}
v_{1} & =a_{2} r_{3}\left(\bar{q}-\bar{q}^{*}\right)+a_{3}\left(\bar{u}-\bar{u}^{*}\right) \\
w_{1} & =\frac{1}{\bar{q}}\left[a_{1}\left(\bar{q}-\bar{q}^{*}\right)+a_{2}\left(\bar{u}-\bar{u}^{*}\right)\right]
\end{aligned}
$$

The control $v$ will be computed using relation (2).

To conclude with, we have found controls $v_{1}, v_{2}$ such that, the closed-loop averaged system (3) can be written as

$$
\dot{\bar{x}}=(J-R) \frac{\partial(V+\Delta V)}{\partial \bar{x}^{T}}
$$

for a new energy function $V_{d}=V+\Delta V$. Actually, $V_{d}(\bar{x})-$ $V_{d}\left(\bar{x}^{*}\right)$ is a local Lyapunov-like function of this system around the equilibrium point $\bar{x}^{*}$.

Taking into account the above conditions, we conclude that $\bar{x}^{*}=\left(\bar{q}^{*}, 0, \bar{u}^{*}\right)$ is an asymptotically stable equilibrium point for the system dynamics (12), and consequently for the dynamics (1). This fact has been achieved by means of a damping injection through the introduction of the matrix $R$, and an energy shaping, via the new energy for the closed-loop system $V_{d}=V+\Delta V$.

Remark 3.2 The averaging theory (see, for example, Theorem 8.3 from Khalil [5]) assures that the stability properties of the averaged system are the same as the original system ones. Both systems have the same equilibrium point. The error presented in the approximation solution for the system given by the solution of the averaged system depends upon the parameter $\varepsilon$ : the smaller $\varepsilon$ is, the smaller the steady-state error is.

\section{Study of the system and the stability region around the equilibrium point}

The closed-loop system stability can be analized from the conditions for the equilibrium $\bar{x}^{*}$ to be a strict local minimum of $V_{d}$ (7)- (9).

From equation (19), the hessian matrix of $V_{d}$ valued in $\bar{x}^{*}$ is

$$
\left(\frac{\partial^{2} V_{d}}{\partial \bar{x}^{2}}\right)_{\bar{x}=\bar{x}^{*}}=\left(\begin{array}{ccc}
-a_{1} & 0 & -a_{2} \\
0 & 1 & 0 \\
-a_{2} & 0 & -\frac{a_{3}}{r_{3}}
\end{array}\right),
$$

then, inequalities (20) guarantee the Hessian (22) to be positive definite.

In order to design constants $a_{1}, a_{2}, a_{3}$, equation (21) has to be taken into account, thus the next inequality is also required

$$
\begin{aligned}
\frac{\cos \bar{u}}{\bar{q}} & +\frac{1}{\bar{q}}\left[a_{1}\left(\bar{q}-\bar{q}^{*}\right)+a_{2}\left(\bar{u}-\bar{u}^{*}\right)\right]> \\
& >\left[a_{2} r_{3}\left(\bar{q}-\bar{q}^{*}\right)+a_{3}\left(\bar{u}-\bar{u}^{*}\right)\right]^{2}
\end{aligned}
$$

\subsection{Study of the range of the constants appearing in the controller design}

The stability domain, in other words, the range of $a_{1}, a_{2}, a_{3}, r_{3}$ which gives the largest stability region around the equilibrium point $\bar{x}^{*}$ will be established from (20) and (23).

Let $\rho_{\bar{q}}, \rho_{\bar{u}}$ be real positive numbers and $\bar{q}, \bar{u}$ such that

$$
\begin{aligned}
& \left|\bar{q}-\bar{q}^{*}\right|<\rho_{\bar{q}} \\
& \left|\bar{u}-\bar{u}^{*}\right|<\rho_{\bar{u}}
\end{aligned}
$$

Then,

$$
\begin{aligned}
& \frac{\cos \bar{u}}{\bar{q}}+w_{1}-v_{1}^{2}> \\
> & \frac{\cos \bar{u}}{\bar{q}}-\frac{1}{\bar{q}}\left[\left|a_{1}\right| \rho_{\bar{q}}+\left|a_{2}\right| \rho_{\bar{u}}\right]-\left(\left|a_{2}\right| r_{3} \rho_{\bar{q}}+\left|a_{3}\right| \rho_{\bar{u}}\right)^{2} \\
> & \frac{\cos \bar{u}}{\bar{q}^{*}+\rho_{\bar{q}}}-\frac{1}{\bar{q}^{*}-\rho_{\bar{q}}}\left[\left|a_{1}\right| \rho_{\bar{q}}+\left|a_{2}\right| \rho_{\bar{u}}\right]- \\
& -\left(\left|a_{2}\right| r_{3} \rho_{\bar{q}}+\left|a_{3}\right| \rho_{\bar{u}}\right)^{2} \\
> & \frac{\cos \left(\bar{u}^{*}-\rho_{\bar{q}}\right)}{\bar{q}^{*}+\rho_{\bar{q}}}-\frac{1}{\bar{q}^{*}-\rho_{\bar{q}}}\left[\left|a_{1}\right| \rho_{\bar{q}}+\left|a_{2}\right| \rho_{\bar{u}}\right]- \\
& -\left(\left|a_{2}\right| r_{3} \rho_{\bar{q}}+\left|a_{3}\right| \rho_{\bar{u}}\right)^{2}
\end{aligned}
$$

and (23) holds if

$$
\begin{aligned}
\frac{\cos \left(\bar{u}^{*}-\rho_{\bar{q}}\right)}{\bar{q}^{*}+\rho_{\bar{q}}}> & {\left[\left|a_{1}\right| \frac{\rho_{\bar{q}}}{\bar{q}^{*}-\rho_{\bar{q}}}+\left|a_{2}\right| \frac{\rho_{\bar{u}}}{\bar{q}^{*}-\rho_{\bar{q}}}\right]+} \\
& +\left(\left|a_{2}\right| r_{3} \rho_{\bar{q}}+\left|a_{3}\right| \rho_{\bar{u}}\right)^{2}
\end{aligned}
$$

Finaly, let us define

$$
\alpha=\frac{\cos \left(\bar{u}^{*}-\rho_{\bar{q}}\right)}{\bar{q}^{*}+\rho_{\bar{q}}}
$$


and choose $a_{1}, a_{2}, a_{3}$ and $r_{3}$ in such a way that

$$
\begin{aligned}
\left|a_{1}\right| \frac{\rho_{\bar{q}}}{\bar{q}^{*}-\rho_{\bar{q}}}+\left|a_{2}\right| \frac{\rho_{\bar{u}}}{\bar{q}^{*}-\rho_{\bar{q}}} & <\frac{\alpha}{2} \\
\left(\left|a_{2}\right| r_{3} \rho_{\bar{q}}+\left|a_{3}\right| \rho_{\bar{u}}\right)^{2} & <\frac{\alpha}{2}
\end{aligned}
$$

then (24) holds.

A possible solution for $a_{1}, a_{2}, a_{3}$ and $r_{3}$ is

$$
\begin{aligned}
& \left|a_{1}\right|<\left|\frac{\alpha\left(\bar{q}^{*}-\rho_{\bar{q}}\right)}{4 \rho_{\bar{q}}}\right| \\
& \left|a_{2}\right|<\left|\frac{\alpha\left(\bar{q}^{*}-\rho_{\bar{q}}\right)}{4 \rho_{\bar{u}}}\right| \\
& \left|a_{3}\right|<\left|\frac{\sqrt{\alpha}}{2 \rho_{\bar{u}} \sqrt{2}}\right| \\
& \left|r_{3}\right|<\left|\frac{\sqrt{\alpha}}{2 a_{2} \rho_{\bar{q}} \sqrt{2}}\right|
\end{aligned}
$$

$>$ From (20) we should consider the constants $a_{1}, a_{3}$ as negative values, the sign of $a_{2}$ can be either positive or negative, and the constant $r_{3}$ is a positive one, since the matrix $R$ is needed to be positive semi-definite. Then, in addition to conditions (25), we will also take into account $a_{1} a_{3}-r_{3} a_{2}^{2}>0$.

Note that from our particular solution of (13) the averaged dynamics becomes linear. One can take benefit from this closing the design process as a pole placement problem.

\section{Simulation results}

Previous results are particularized to $x^{*}=(0.5,0,1.2), \rho_{\bar{q}}=1$, $\rho_{\bar{u}}=0.5$. Then $a_{1}, a_{2}, a_{3}$ and $r_{3}$ has been chosen as $a_{1}=$ $-0.05, a_{2}=-0.11, a_{3}=-0.48, r_{3}=1.8$.

Figures 2, 3, 4 and 5 display the dynamics of system (1), actually, the trajectories of the states, the control $v$, the auxiliar controls $v_{1}$ and $v_{2}$ and the new energy, respectively. Initial conditions are $(0,0,0)$ and $\varepsilon=0.02$

\section{Conclusions}

In this paper, we have locally stabilized the variable-length pendulum around an equilibrium point by means of an energy approach. The passivity-based control design methodology of Energy Shaping and Damping Injection is applied, and a study of the coefficients appearing in the obtained controller in connection to the closed-loop system stability is provided.

The closed-loop system obtained has a well-defined energy structure in which it is distinguished a conservative and a dissipative components derived from the new closed-loop system energy. Therefore, the proposed stabilization method is a Lyapunov-based design where the construction of the closedloop system Lyapunov function which ensures the system stability is based upon the ESDI methodology.

The results here obtained can be completed by analyzing the stability region of the resulting controlled system, which will lead us to global stability results. A study of stability depending on the values for $\rho_{\bar{q}}$, and $\rho_{\bar{u}}$ is required.

It will also be interesting to extend the stabilization problem to solve the tracking problem, for which we may use timedepending Lyapunov-like functions.

\section{Figures}
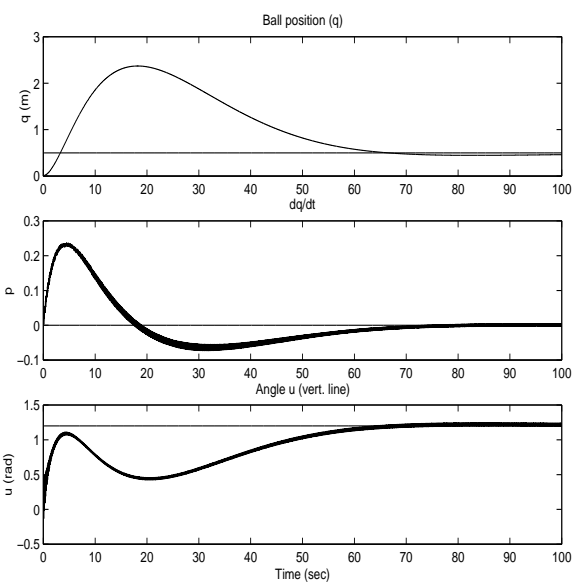

Figure 2: State variables.

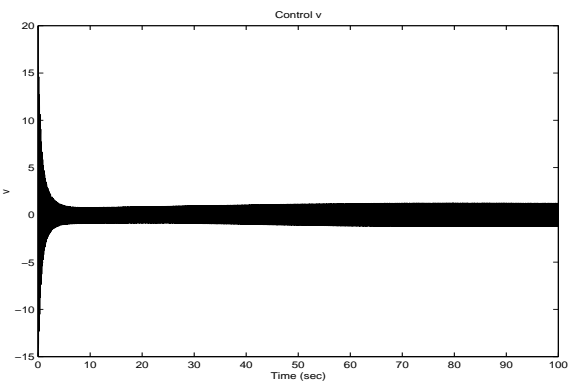

Figure 3: Control v.
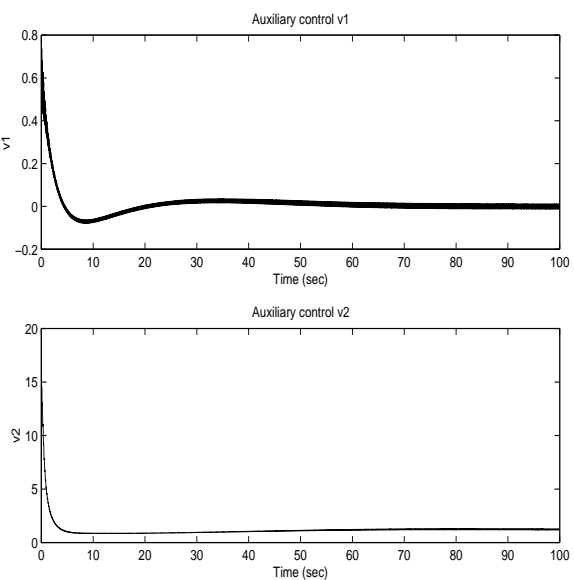

Figure 4: Auxiliary controls $v_{1}, v_{2}$. 


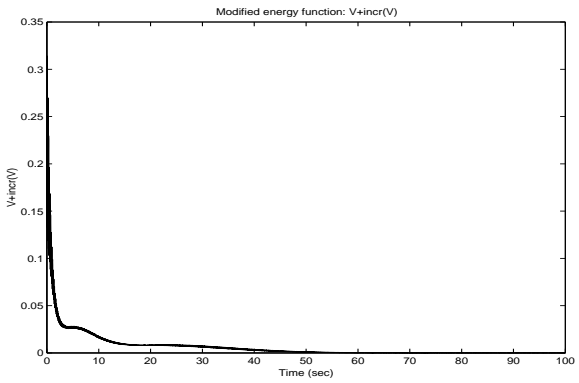

Figure 5: Modified energy $V+\Delta V$.

\section{Acknowledgement}

Work partially supported by CICYT under Grant n.TAP97C03-0969-01

\section{References}

[1] C. I. Byrnes, A. Isidori, J. C. Willems. "Passivity, feedback equivalence, and the global stabilization of minimum phase nonlinear system", IEEE Trans. on Aut. Control, 36, pp. 1228-1240, (1991).

[2] A. Bressand, F. Rampazzo. "On differential systems with quadratic impulses and their applications to lagragian dynamics", SIAM Journal on Control and Optimization, 31, pp. 1205-1220, (1993).

[3] M. Fliess, J. Lévine, P. Martin, P. Rouchon. "Flatness and defect of non-linear systems: introductory theory and examples", International Jorurnal of Control, 61(6), pp. 1327-1361, (1995).

[4] R. Kelly. "Regulation of manipulators in generic task space: an energy shaping plus damping injection approach", IEEE Transactions on Robotics and Automation, 15(2), pp. 381-386, (1999).

[5] K. Khalil. Nonlinear Systems (Prentice-Hall, 1996).

[6] B. M. J. Maschke, R. Ortega, A. J. van der Schaft. "Energy-based Lyapunov functions for forced Hamiltonian systems with dissipation", Proceedings fo the 37th Conference on Decision and Control, pp. 3599-3604, (1998).

[7] P. J. Nicklasson, R. Ortega, G. Espinosa-Pérez. "Passivity-based control of a class of Blondel-Park transformable electric machines", Modeling, Identification and Control, 18(4), pp. 273-305, (1997).

[8] R. Ortega, A. Loria, R. Kelly, L. Praly. "On passivitybased output feedback global stabilization of EulerLagrange systems", Int. J. Robust and Nonlinear Control, 5, pp. 313-324, (1995).
[9] R. Ortega, Z. P. Jiang, D. J. Hill. "Passivity-based control of nonlinear systems: a tutorial", Proceedings of the American Control Conference, pp. 2633-2637, (1997).

[10] R. Ortega, A. Loria, P. J. Nicklasson, H. Sira-Ramírez. Passivity based Control of Euler Lagrange Systems. Mechanical, Electrical and Electromechanical Applications (Springer-Verlag, London, 1998).

[11] R. Ortega, A. J. van der Schaft, B. Maschke, G. Escobar. "Energy-shaping of port-controlled Hamiltonian systems by interconnection", Proceedings of the 38th Conference on Decision and Control, pp. 1646-1651, (1999).

[12] H. Sira-Ramírez, R. A. Pérez Moreno, R. Ortega, M. García Esteban. "Passivity-based controllers for the stabilization of DC-t-DC power converters", Automatica, 33(4), pp. 499-513, (1997).

[13] H. Sira-Ramírez. "A general canonical form for feedback passivity", International Journal of Control, Special Issue: Recent Advances in the Control of Nonlinear Systems, 71(5), pp. 891-905, (1998).

[14] H. Sira-Ramírez. "On the control of the variable length pendulum", Proceedings of the 38th IEEE Conference on Decision and Control, pp. 1188-1189, (1999).

[15] H. Sira-Ramírez, E. M. Navarro-López. "On the passivity of general nonlinear systems", Preprint International Symposium on Mathematical Theory of Networks and Systems (Perpignan, France), (2000). 